\title{
Balkanologie
}

Balkanologie Revue d'études pluridisciplinaires

Vol. I, n 1 | 1997

Volume I Numéro 1

\section{Serbie : une transition démocratique inachevée}

\section{Yves Tomić}

\section{OpenEdition}

\section{Journals}

Édition électronique

URL : http://journals.openedition.org/balkanologie/209

DOI : 10.4000/balkanologie.209

ISSN : 1965-0582

\section{Éditeur}

Association française d'études sur les Balkans (Afebalk)

\section{Édition imprimée}

Date de publication : 1 juillet 1997

ISSN : 1279-7952

\section{Référence électronique}

Yves Tomić, «Serbie : une transition démocratique inachevée », Balkanologie [En ligne], Vol. I, n 1 | 1997, mis en ligne le 02 juin 2008, consulté le 17 décembre 2020. URL : http://

journals.openedition.org/balkanologie/209; DOI : https://doi.org/10.4000/balkanologie.209

Ce document a été généré automatiquement le 17 décembre 2020.

(c) Tous droits réservés 


\title{
Serbie : une transition démocratique inachevée
}

\author{
Yves Tomić
}

1 Le vol de la victoire de la coalition Zajedno (Ensemble) aux élections municipales de Serbie par le Parti socialiste dominant en novembre 1996 a provoqué la formation d'un imposant mouvement civique dans le pays. La mobilisation a affecté les grands centres urbains, industriels et intellectuels (Belgrade, Niš, Novi Sad, Kragujevac, Kraljevo, etc.) ainsi que les plus petites villes (Pirot, Čačak, Gornji Milanovac, Loznica, etc.)1. Régulièrement, les citoyens dépossédés de leur voix ont marché dans le centre des villes pour manifester leur mécontentement et pour exiger le respect de leur volonté politique exprimée lors de la dernière consultation électorale. Pour la première fois depuis 1990, le pouvoir n'est pas parvenu à enrayer le mouvement de contestation et à sortir rapidement de la crise. Par ailleurs, les principaux partis de l'opposition démocratique (Mouvement du renouveau serbe - Srpski Pokret Obnove -, Parti démocrate - Demokratska Stranka - et Alliance civique de Serbie - Gradjanski Savez Srbije -) ont compris que par leur union ils pouvaient rivaliser sérieusement avec les socialistes.

\section{La transformation idéologique de la Ligue des communistes de Serbie}

Du fait de la guerre ayant éclaté en 1991 et de son implication directe ou indirecte, la Serbie n'a pas été affectée par les transformations qui ont eu cours dans la plupart des pays ex-communistes d'Europe centrale et orientale: malgré l'établissement du pluralisme politique, sa transition à la démocratie n'est pas achevée, les entraves à la liberté d'expression étant encore nombreuses, en raison notamment du monopole du pouvoir sur les grands médias; le régime de Slobodan Milošević ne s'est pas encore engagé sur la voie des réformes économiques nécessaires à la revitalisation de l'appareil productif. Quelles sont les chances de la Serbie de réintégrer l'Europe ? L'opposition peut-elle venir à bout du pouvoir socialiste en Serbie? Afin de répondre à ces questions, il n'est pas inutile de revenir sur les développements de la vie politique 
en Serbie depuis 1987, année de la transformation idéologique de la Ligue des communistes, de l'adoption de l'idéologie nationale par cette dernière.

Le Parti socialiste serbe (SPS - Socijalistička Partija Srbije), héritier de la Ligue des communistes de Serbie, a opéré depuis 1994-1995 un changement de sa source de légitimité en mettant l'accent sur la nécessité d'aboutir à la paix et de se concentrer sur le développement économique de la Serbie: depuis 1987, les communistes, puis les socialistes, avaient mis au premier plan la défense des intérêts nationaux du peuple serbe. La récupération, à partir de 1987, et l'exploitation du mouvement de contestation des Serbes et Monténégrins du Kosovo, appuyés par une grande partie des intellectuels serbes et l'Église orthodoxe, lui avait procuré une nouvelle source de légitimité, alors que le pays sombrait dans une profonde crise économique et sociale. Tandis que la plupart des Partis communistes s'épuisaient, aussi bien dans le cadre de la fédération yougoslave que dans les pays du bloc soviétique, la Ligue des communistes de Serbie parvint à sortir de la léthargie et à se redynamiser en s'appuyant sur l'idéologie nationale serbe ${ }^{2}$. Entre 1987 et 1989, les milieux intellectuels d'opposition soutinrent directement ou indirectement le pouvoir communiste qui exploita leurs idées: Mémorandum de l'Académie des sciences et des arts de 1986, etc. La rupture entre les intellectuels d'opposition et le pouvoir se produisit au cours de l'année 1989, au fur et à mesure du recul des élites communistes en Europe centrale et orientale.

4 Cette mutation idéologique du communisme au nationalisme sous une forme populiste permit aux communistes serbes de se maintenir au pouvoir lors de l'effondrement général du système communiste en Europe centrale et orientale. En opérant le choix de la nation dès 1987, ils devancèrent l'opposition qui allait se constituer essentiellement autour de cette thématique. En 1990, lors de la tenue des premières élections libres depuis 1945, la Ligue des communistes de Serbie, transformée en Parti socialiste, se présenta avec des résultats positifs dans son combat pour la défense des intérêts nationaux : les communistes étaient en effet parvenus à supprimer les autonomies des provinces de Voïvodine et du Kosovo et à recentraliser leur État, l'élément déterminant fut la reprise en main du pouvoir dans la province autonome du Kosovo où les Serbes se déclaraient victimes d'un génocide, en fait, de pressions de la part des Albanais visant à les faire quitter cette région ${ }^{3}$.

\section{La domination du Parti socialiste (ex-communiste)}

5 Lors des élections de décembre 1990, le Parti socialiste serbe s'assura une position dominante grâce au contrôle des principaux médias, de l'appareil d'État, des entreprises sociales. Il fixa lui-même les règles du jeu politique et repoussa au maximum la tenue du premier scrutin pluraliste. Avant l'organisation de ce dernier, il modifia la Constitution en fonction de ses propres intérêts: celle-ci augmenta significativement les prérogatives du président de la République au détriment du Parlement ${ }^{4}$. De la sorte, S. Milošević, se sachant favori aux élections présidentielles, pouvait préserver l'essentiel du pouvoir en cas de défaite de sa formation politique aux élections législatives. Critiqués par les partis de l'opposition nationaliste extrémiste, et, plus particulièrement, par le Mouvement du renouveau serbe (SPO) de Vuk Drašković, les socialistes serbes se présentèrent comme les garants de la paix civile. L'opposition à l'époque se divisait en deux principaux courants: l'un national représenté par le Mouvement du renouveau serbe, l'autre libéral et civique représenté par le Parti 
démocrate, le Parti réformiste etc. ${ }^{5}$ Les socialistes obtinrent $77,6 \%$ des sièges au Parlement alors qu'ils avaient obtenu $45,8 \%$ des voix, le SPO 7,2\%, le Parti démocrate $2,8 \%$. L'opposition n'était pas parvenue à s'unir contre les socialistes en raison des différences de programmes et des rivalités de ses chefs. Elle ne se regroupa que lors du second tour alors qu'il était déjà trop tard. Cette union (Opposition démocratique unie de Serbie) fut d'ailleurs de courte durée.

6 Ayant conscience que le contrôle des médias par les socialistes avait contribué à sa défaite, le SPO organisa une manifestation le 9 mars 1991 afin d'obtenir la démocratisation des principaux médias dont la télévision. La police finit par intervenir, le rassemblement ayant été interdit. La manifestation se transforma de ce fait en bataille de rues. Paniqué par l'ampleur de la manifestation, le pouvoir appela l'armée en renfort. Face à la répression exercée par les autorités, les étudiants sortirent dans la rue le 11 mars afin d'apporter leur soutien aux revendications démocratiques et les autres partis de l'opposition participèrent au mouvement de contestation. Toutefois, ces manifestations ne virent pas la constitution d'un bloc oppositionnel solide. Les partis continuaient à vouloir mener seuls leur lutte politique soucieux avant tout de préserver leur identité. Le pouvoir dut provisoirement reculer. À cette époque, la crise de la fédération yougoslave atteignit son paroxysme avec la tentative des dirigeants serbes de proclamer l'état d'exception sur tout le territoire yougoslave. L'éclatement de la guerre en Slovénie, puis, surtout, en Croatie, limita encore plus l'influence des partis d'opposition. Paradoxalement le Mouvement du renouveau serbe ne soutint pas la politique belliciste de la Serbie, mais appuya le mouvement antiguerre naissant. Ce revirement provoqua la chute du nombre de ses membres et partisans. Cette diminution profita au Parti radical serbe - Srpska Radikalna Stranka - de Vojislav Šešelj qui avait décidé de collaborer avec le régime de S. Milošević.

\section{Les luttes politiques en Serbie : 1992-1996}

7 En 1992, après la signature des accords de paix en Croatie en janvier, l'éclatement de la guerre en Bosnie en avril, l'opposition au pouvoir s'accentua à travers la création du Mouvement démocratique de Serbie - DEPOS - au mois de mai sur la proposition d'intellectuels. La légitimité du régime de Belgrade tomba au plus bas avec l'expression du mécontentement des principales institutions culturelles (universités, Académie des sciences, etc.) ainsi que de l'Église orthodoxe serbe. Au mois de juin les étudiants entamèrent une grève, la contestation s'élargit dans les rues de Belgrade sans débordement du 28 juin au 5 juillet, les rassemblements attirant parfois plusieurs centaines de milliers de personnes. Toutefois, l'opposition ne parvint pas à obtenir la démission de S. Milošević et la formation d'un gouvernement de transition. Cette vague de contestation du mois de juin 1992 fut la plus importante de la période 1990-1995.

8 Le pouvoir serbe parvint à se sortir de la crise en faisant appel à des personnes extérieures aux rangs du Parti socialiste : Milan Panić, riche industriel vivant aux ÉtatsUnis d'Amérique, fut invité début juillet 1992 à composer un nouveau gouvernement fédéral à l'initiative de Dobrica Ćosić, élu à la présidence de la fédération serbomonténégrine le 15 juin 1992. Les initiatives politiques et diplomatiques de M. Panić et de D. Ćosić furent prises en toute indépendance du Parti socialiste serbe, ce qui provoqua des conflits entre eux et l'élite dominante. Dès le 31 août, le Parti radical serbe, allié des socialistes, posa la question de confiance au gouvernement fédéral. Ce 
dernier parvint à se maintenir lors de votes de confiance le 4 septembre, puis les 2 et 3 novembre. Lors de nouvelles élections générales (fédérales, républicaines et locales) le 20 décembre 1992, le Parti socialiste sortit vainqueur en obtenant $40,4 \%$ des sièges du Parlement (101 sièges pour 28,77 \% des voix) de la République de Serbie. Le Parti radical serbe de V. Šešelj s'imposa comme la seconde force politique du pays $(22,58 \%$ des voix soit $29,2 \%$ des sièges au Parlement de Serbie - 73 sièges -). Le Mouvement démocratique serbe (SPO, Parti démocrate de Serbie) n'obtint que $16,9 \%$ des voix, soit $20 \%$ des sièges (49). Le Parti démocrate qui refusa d'entrer dans la coalition du DEPOS n'obtint que 7 sièges de députés. S. Milošević remporta les élections présidentielles dès le premier tour contre M. Panić en obtenant $56 \%$ des voix (soit 39,67\% de l'ensemble du corps électoral de Serbie) contre $34,02 \%$ pour son rival américano-serbe. Une fois de plus, l'élite au pouvoir parvint à surmonter la crise de légitimité qui l'affectait. Toutefois, le Parti socialiste ne possédait plus, comme entre décembre 1990 et décembre 1992, l'imposante majorité des sièges au Parlement, mais, grâce à l'appui des radicaux de Šešelj, il contrôlait cette institution. Le principal thème de la campagne fut celui de la question nationale serbe sur lequel insistèrent plus particulièrement le Parti socialiste et le Parti radical. Les sanctions internationales contre la Serbie contribuèrent à l'affirmation de l'expression du nationalisme par le pouvoir ainsi qu'au maintien de ce dernier. Le Mouvement démocratique serbe insista sur la nécessité de l'alternance, le Parti démocrate sur la réorganisation du système économique ${ }^{6}$. Le 29 décembre 1992, à l'initiative du Parti radical serbe, le Parlement fédéral vota contre le gouvernement Panic qui dut être remplacé.

9 L'année 1993 fut caractérisée par l'éclatement de l'entente entre le Parti socialiste serbe et le Parti radical serbe qui se produisit après l'acceptation du plan de paix Vance-Owen par le Président serbe S. Milošević à la fin du mois d'avril. Ce fut d'ailleurs à partir de cette époque que le Président serbe opéra un revirement dans sa politique nationale et devint plus conciliant vis-à-vis des propositions des grandes puissances. Ce changement d'orientation se produisit au moment où la Serbie connaissait de graves difficultés économiques et était affectée par l'hyperinflation.

Le $1^{\mathrm{er}}$ juin, l'Assemblée fédérale retira sa confiance au président yougoslave D. Ćosić. À cette occasion, plusieurs milliers (de 3000 à 5000 ) de personnes manifestèrent à l'initiative du Mouvement du renouveau serbe contre cette mesure. Le rassemblement dégénéra (un policier fut tué) et les principaux dirigeants de ce mouvement, dont Vuk et Danica Drašković, furent arrêtés. Les réactions de l'opposition (SPO et Alliance civique essentiellement, les autres partis - Parti démocrate, Parti démocrate serbe, etc. étant opposés à la politique nationale des premiers et soutenant le chef de la "République serbe" de Bosnie, Radovan Karadžić) furent limitées et seules quelques manifestations furent organisées, regroupant quelques milliers d'individus notamment les 19, 26 juin et 4 juillet. Vuk et Danica Drašković furent relâchés le 9 juillet 1993 grâce aux pressions internationales exercées sur le pouvoir serbe.

11 À l'automne, le conflit entre le Parti socialiste serbe et le Parti radical s'accentua lorsque les députés radicaux exigèrent en octobre un vote de confiance au gouvernement de Nikola Sainović. En fait, il ne s'agissait pas tant pour les radicaux de dénoncer la politique intérieure du gouvernement que la politique extérieure de $\mathrm{S}$. Milošević. Dans ces circonstances, ce dernier décida de dissoudre l'Assemblée le 20 octobre et d'organiser de nouvelles élections. Le 19 décembre 1993, les troisièmes élections législatives en l'espace de 4 ans eurent lieu. Le Parti socialiste en sortit encore 
une fois vainqueur et obtint 123 sièges sur 250. Le Mouvement démocratique serbe (Mouvement du renouveau serbe, Alliance civique, Nouvelle démocratie - Nova Demokratija -) conquit 45 sièges, les radicaux 39, le Parti démocrate 29, le Parti démocrate serbe 7, etc.

12 En 1994, le Parti socialiste confirma son revirement en faveur de la paix en adoptant le plan de paix du "groupe de contact" que les dirigeants serbes de Bosnie rejetèrent. Face à la résistance de ces derniers, le pouvoir de Belgrade choisit de rompre ses relations politiques et économiques avec la "République serbe de Bosnie" et d'établir un embargo à son égard. L'évolution des positions des socialistes au pouvoir ainsi que de la situation en Bosnie-Herzégovine ne furent pas sans répercussion sur l'échiquier politique serbe: le Parti radical serbe maintint son opposition à la politique de $\mathrm{S}$. Milošević, le considérant comme un traître à la cause nationale. Le Parti radical fut rejoint en cela plus fermement par le Parti démocrate dirigé par Zoran Djindjić et le Parti démocrate serbe de Vojislav Koštunica. À l'opposé, le Mouvement du renouveau serbe et l'Alliance civique furent amenés à appuyer sans compromission le nouveau cours du président serbe.

13 Entre 1990 et 1993-1994, du fait de la guerre en Bosnie, les positions de certains partis politiques évoluèrent sensiblement: le Mouvement du renouveau serbe passa d'un programme nationaliste extrémiste à une critique véhémente de la politique de guerre menée par S. Milošević sans toutefois abandonner des référents nationaux (monarchie, mouvement de résistance anticommuniste entre 1941 et 1945 (Tchetniks) - Ravnogorski Pokret -) et sans renier l'objectif de la création d'un État unique des Serbes formulé dans son programme de 1994 en des termes plus modernes ${ }^{7}$. Autre cas significatif, celui du Parti démocrate qui du centre s'engagea vers la droite et insista de plus en plus sur les intérêts nationaux du peuple serbe. Il en fut de même du Parti démocrate de Serbie, produit d'une scission au sein du Parti démocrate, en raison du refus de ce dernier d'entrer en 1992 dans le Mouvement démocratique serbe - DEPOS -. En 1993, ce parti modifia ses positions sur la question nationale en approuvant et défendant la politique de R. Karadžić en Bosnie. En outre, il faut souligner que la plupart des partis politiques en 1990 s'entendaient sur la nécessité de créer un État unitaire des Serbes en cas de désintégration de la fédération yougoslave. Il n'existait pas de programme national alternatif à celui du pouvoir qui était, en fait, celui d'une grande partie de la société. Il est important de rappeler que les partis politiques d'opposition furent créés, en ce qui concerne leurs noyaux dirigeants, par les intellectuels qui dans la seconde moitié des années 1980 ouvrirent la question nationale serbe. Ce consensus autour de la question nationale ne fut finalement pas remis sérieusement en cause et ne favorisa pas la constitution d'une réelle opposition au régime de S. Milošević. Au contraire, une concurrence se développa entre le parti dominant et un grand nombre de partis pour savoir qui représentait et défendait le mieux les intérêts nationaux. Seule l'Alliance civique fondée en novembre 1992 (rassemblement de plusieurs organisations : Union des forces réformistes, Club républicain, etc.) se démarqua par son opposition au nationalisme, mais elle fut et demeure encore une force politique marginale de par son poids électoral. 


\section{Conclusion} politique de paix par le Mouvement du renouveau serbe et l'Alliance civique / formation d'un bloc nationaliste par le Parti radical, le Parti démocrate et le Parti démocrate serbe) qui s'amorça à partir d'avril 1993 et de l'acceptation du plan VanceOwen et se renforça en 1994, fut opératoire jusqu'en novembre 1995, à savoir jusqu'aux négociations de Dayton. À l'automne, le Parti socialiste serbe écarta en plusieurs étapes de sa direction les membres critiquant le revirement du président serbe et défendant la cause nationale en Bosnie-Herzégovine: Milorad Vučelić, Zivorad Minović, Borisav Jović, Mihailo Marković, l'idéologue de l'organisation, Radovan Pankov. Il se déclara prêt à entrer en coalition avec la Gauche unie yougoslave - Jugoslovenska Ujedinjena Levica -, fondée en mars 1995 et regroupant plusieurs dizaines d'organisations et mouvements communistes, où la femme de Milošević joue un rôle influent. les accords de paix et la fin de la guerre en Bosnie quand bien même ils les trouvassent injustes. De la même façon que le Parti socialiste, les discours politiques se recentrèrent sur la vie économique et la nécessité de réintégrer le monde et l'Europe en particulier. Toutefois, le parti dominant ne semblait pas disposer à avancer sur la voie des changements économiques et d'une réelle démocratisation. Les partis d'opposition soulevèrent de nouveau la question de leur union afin de faire échec au Parti socialiste lors des consultations électorales à venir. Trois blocs se constituèrent : le premier ayant pour nom Alliance démocratique rassembla le Parti démocrate s'étant débarrassé de Dragoljub Mićunović, premier président du Parti, et de ses partisans, qui avaient dénoncé la dérive nationaliste du chef du parti, Z. Djindjić, le Parti démocrate serbe de V. Koštunica, le Parti de l'assemblée populaire - Saborna Narodna Stranka -, produit d'une scission du Mouvement du renouveau serbe en 1994, le Parti libéral serbe - Srpska Liberalna Stranka - ; le second, sous la dénomination de "centre démocratique" regroupa le SPO, l'Alliance civique, le Parti paysan populaire, etc., le troisième bloc étant constitué du seul Parti radical de V. Šešelj qui refusait de participer à toute coalition. L'ensemble de ces partis se retrouvèrent unis le 26 décembre 1995 dans leur volonté d'établir des institutions réellement démocratiques à travers la formation d'un parlement parallèle réunissant la totalité des partis d'opposition ayant des députés à l'Assemblée de Serbie, y compris les partis des minorités (Communauté démocratique des Hongrois de Voïvodine, Parti pour l'action démocratique représentant les Albanais des villes de Preševo, Bujanovac et Medvedje). Cette initiative fut prise notamment pour protester contre la suppression de la retransmission des débats parlementaires à la télévision d'État (RTS) décidée lors de la session du printemps 1995. Cette union de l'opposition ne pouvait tenir qu'autour d'exigences minimales (vaincre les socialistes aux élections, réformes économiques marchandes) tant les disparités idéologiques concernant la question nationale étaient importantes.

La tentative de créer un Parlement parallèle échoua, les partis d'opposition se dispersèrent à nouveau. Toutefois, trois partis prirent conscience de l'indispensable unité pour vaincre les socialistes : le Mouvement du renouveau serbe de V. Drašković, l'Alliance civique de Vesna Pešić et le Parti démocrate de Z. Djindjić. Ces trois partis, bien qu'ayant adopté des positions divergentes quant à la guerre en Bosnie, se rapprochèrent en février-mars 1996 et décidèrent de célébrer ensemble les 
manifestations du 9 mars 1991. Ils organisèrent par la suite une série de meetings dans les grandes villes de Serbie afin de préparer leur campagne pour les élections fédérales. L'activisme de ces trois partis depuis le printemps 1996, et malgré une crise interne en août 1996, s'avéra payant. Ils se retrouvèrent à la tête du mouvement de contestation du pouvoir de $\mathrm{S}$. Milošević après le 17 novembre 1996.

Le formidable mouvement de révolte contre le vol électoral orchestré par le pouvoir a révélé avec acuité la réelle nature du régime de $\mathrm{S}$. Milošević. Six ans après la chute des régimes communistes en Europe centrale et orientale, la Serbie a connu un mouvement civique similaire à ceux de Berlin-est ou Prague. Cette fois-ci, il ne s'agissait plus de définir les autres (à savoir les non-Serbes) comme coupables mais bien les dirigeants serbes autoritaires ayant conduit leurs co-nationaux vers la guerre, l'appauvrissement et l'isolement international de leur pays.

\section{NOTES}

1. Cf. carte de la contestation publié dans l'hebdomadaire Vreme du 14/12/96, p. 9.

2. Cf. Tomić (Yves), «Milosevic et la mutation de la Ligue des communistes de Serbie: du communisme au nationalisme populiste », Autre Europe (L'), (34-35), 1996.

3. Goati (Vladimir), Jugoslavija na prekretnici : od monizma do gradjanskog rata (La Yougoslavie au tournant: du monisme à la guerre civile), Beograd : Jugoslovenski institut za novinarstvo, 1991, p. 33.

4. La Constitution fut adoptée par le Parlement en septembre 1990 alors qu'il était encore composés des seuls socialistes (ex-communistes). La loi électorale fut également élaboré par le seul Parti dominant. Le Président se voyait donner la possibilité d'opposer son veto aux lois établies par le Parlement et le gouvernement sans l'avis préalable du Premier ministre. Samardžić (Slobodan)., Prinudna zajednica i demokratija (Communauté coercitive et démocratie), Beograd : Akademia nova / Institut za evropske studije, 1994, pp. 188-190.

5. Antonić (Slobodan), "Promene stranačkog raspoloženja gradjana Srbije 1990-1993 », in Antonić (Slobodan), Jovanović (Milan), Marinković (Darko), ur., Srbija izmedju populizma $i$ demokratije : politički procesi u Srbiji 1990-1993 (La Serbie entre populisme et démocratie : processus politiques en Serbie, 1990-1993), Beograd : Institut za političke studije, 1993, pp. 13-14.

6. Slavujević (Zoran)., « Borba za vlast u Srbiji kroz prizmu izbornih kampanja » (La lutte pour le pouvoir en Serbie à travers le prisme des campagnes électorales), in Goati (Vladimir), Slavujević (Zoran), Pribićević (Ognjen), ur., Izborne borbe u Jugoslaviji 1900-1992 (Les luttes électorales en Yougoslavie, 1990-1992), Beograd, 1993, pp. 136-140.

7. Stojanović (Dubravka)., "Traumatični krug srpske opozicije» (Le cercle traumatique de l'opposition serbe), Ogledi (suppl. de Republika), (125-126), 1-31/10/1995, p. x. 


\section{RÉSUMÉS}

Depuis leur formation, les partis politiques en Serbie ont modifié leurs programmes et leur idéologie. Tout comme la Ligue des Communistes de Serbie qui, en 1987, prenait la défense des intérêts nationaux serbes et, en 1993, commença à soutenir les propositions internationales de règlement des conflits en Bosnie-Herzégovine. Toutefois, l'articulation demeurait fondée autour de la question nationale serbe. Les manifestations de novembre 1996 marquent, de ce point de vue, un tournant: les partis d'opposition accusent désormais les dirigeants serbes d'être responsables de l'appauvrissement et de l'isolement international de la Serbie.

Since their creation in 1990 , the political parties in Serbia have modified their programs and their ideology. Juste like the Serbian Communist Ligue which, in 1987, championed the Serbian national interests, and, in 1993, began to support the proposals of the international community to put an end to conflicts in Bosnia and Herzegovina. Yet, the political field was structured on the Serbian national question. From this point of view, the demonstrations of november 1996 point out a revolution : from now on, the political opposition accuse the rulers of being responsible for the empoverishment and the international isolation of Serbia.

\section{AUTEUR}

\section{YVES TOMIĆ}

Yves Tomić est responsable des secteurs ex-yougoslave et bulgare de la Bibliothèque de documentation internationale contemporaine. 